\title{
Test and measurements of reliability performance on radioreceiver chains for the Northern Cross Radio Telescope ${ }^{\star}$
}

\author{
M. Catelani ${ }^{1}$, V. L. Scarano ${ }^{1}$, S. Montebugnoli ${ }^{2}$, G. Bianchi ${ }^{2}$, and F. Perini ${ }^{2}$ \\ 1 University of Florence, via S. Marta 3, 50139, Florence, Italy \\ email: marcantonio.catelani@unifi.it, valeria.scarano@unifi.it \\ 2 I.N.A.F. - I.R.A, Via Fiorentina, 3508/B 40059 Medicina, Italy \\ email: s.montebugnoli@ira.inaf.it,g.bianchi@ira.inaf.it,f.perini@ira.inaf.it
}

\begin{abstract}
Reliability analysis of two radioreceiver chains architecture were performed in order to choose the solution with the highest value of Mean Time Between Failures and minimize faults in the system, with the consequent lower maintenance costs. Located the most stressed devices from a climatic-environmental point of view, we decided to monitor their real thermal solicitation in order to evaluate the proper reliability performances varying temperature and the possible degradation of the critical subsystem and consequently of the entire chain. The design for reliability approach proposed in this research for the reinstrumentation of the Northern Cross Radiotelescope could be an useful aid for the design of the international SKA Telescope. Since it will be extremely large (1 square kilometre) the maintenance costs, related to the reliability, become an important parameter to evaluate. This research activity was developed in partnership with the Istituto di Radioastronomia, Istituto Nazionale di Astrofisica, in the context of the Square Kilometer Array Design Study supported by UE FP6.
\end{abstract}

\section{Introduction}

This research activity was developed in collaboration with Istituto di Radioastronomia, Istituto Nazionale di Astrofisica (IRA-INAF), in the BEST project (Basic Element for SKADS Training) (Montebugnoli et al. 2009), that allowed to the reinstrumentation of the Northern Cross in Medicina, Bologna, Italy (Montebugnoli et al. 2004). The aim was to develop new technologies and algorithms for application in the Square Kilometer Array Design Study (SKADS) project (van Ardenne et al. 2000). Studies have been made for the implementation of the radio telescope called Square Kilometre Array (Dewdney et al. 2009) that will lead the onset of the next years. This instrument will image celestial radio sources based on the principle of earth rotation synthesis. In last year of the project it concentrated on the science questions made possible by its orders of magnitude increase in sensitivity together with a large field of view, three decades frequency coverage and large resolution range among others. Various countries agreed to the project and many aspects of great importance the technical implementation of SKA were studied. These aspects covered new antenna concepts and new and enabling technologies in fields ranging from broadband antennas, low noise (integrated) frontends, photonics, signal processing and packaging in electronic industry to calibration, interference mitigation strategies and image processing, concept design and architecture (Dewdney et al. 2009). Here, we will emphasise the approaches of the design for reliability in order to compare two different radioreceiver chains architecture applied to the Northern Cross, these studies are fundamental also for SKA technologies in view of

^ This work was supported by the European Commission Framework Program 6, Project SKADS, Square Kilometre Array Design Studies (SKADS), contract no 011938. some important system requirements. This kind of approach has never been applied in radio astronomy. Although highly ambitious in scope and desired performance, SKA at the same time relies on technologies and trends because of needs in other areas e.g. in wireless telecommunications and in general ICT applications. The SKA system will be characterized by an effective collecting area of one square kilometre, an area more than 30 times grater than the largest telescope, with the aim to provide increase in sensitivity with respect to the actual solutions (Hall et al. 2004). The place for the SKA Radiotelescope could be in South African desert or in Australian one; any way independently where SKA will be located, the environmental conditions play a fundamental role in both the reliability and availability performances of the radiotelescope. To this aim, a reliability studies and results on the performances of BEST project (Bolli et al. 2008), test bed for SKA, is presented in this work. The paper is structured as follows. After a brief presentation of the Northern Cross radiotelescope, the paper focuses the attention on the investigation of two different radioastronomical signal receiver chain architectures reliability performance. The solution with the highest Mean Time Between Failure (MTBF) is really implemented and, for this critical subsystem, a deep analysis by the environmental stress, in particular temperature, was done.

\section{Technological design and choices}

In this section we present a brief description of the receiver chain implemented into the Northern Cross radiotelescope. The system, located at Medicina (Bologna-Italia), is composed by a North-South arm and an East-West arm. In order to reinstrument the radiotelescope two different solutions of radioastronomical signal receiver chains are presented in the fol- 


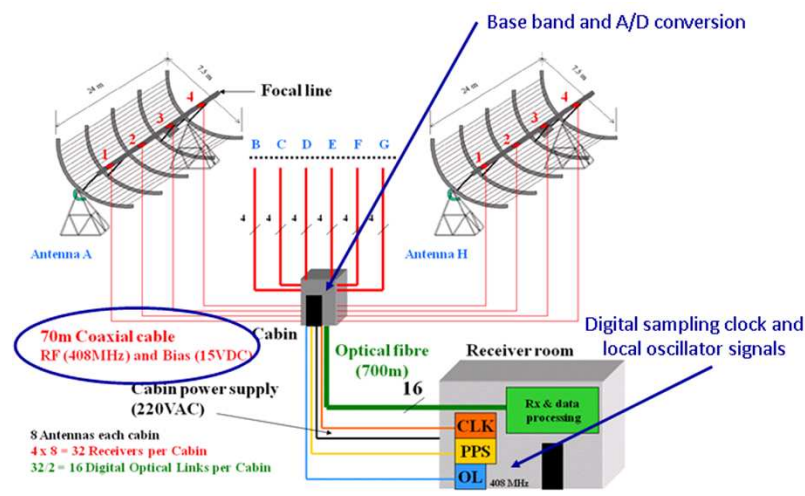

Fig. 1: Digital Optical Link Architecture

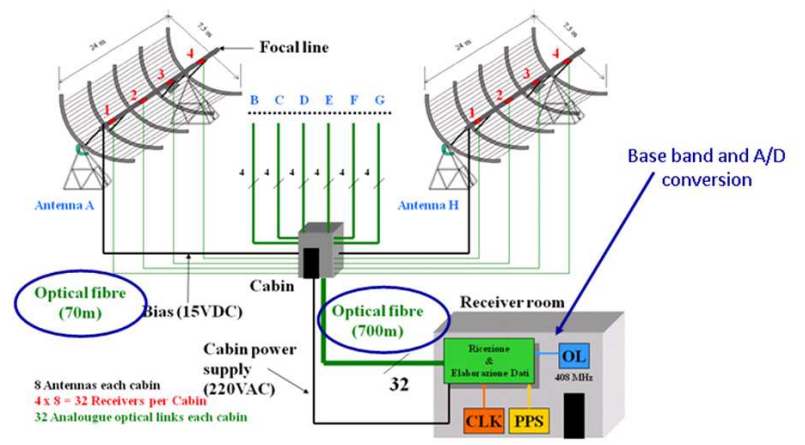

Fig. 2: Analogue Optical Link Architecture

lows. Describing the digital optical link and analogue optical link solutions, only 8 cylindrical reflectors of the N-S arm are considered for sake of simplicity. In particular for each cylindrical reflector, named Antenna from $\mathrm{A}$ to $\mathrm{H}$, there are four receivers $(1-4)$ placed on the focal line of the antenna.

The first solution, named digital optical link (see in Fig. 1), shows the path of the signal transmission and control. As we can observe, the astronomical signal is received by an array of dipoles and sent to a front-end stage composed by a low noise amplifier, a pass-band filter, with $408 \mathrm{MHz}$ as central frequency and $16 \mathrm{MHz}$ of bandwidth, and one amplifier stage. The signal filtering is necessary in order to remove the unwanted interferences, while the low noise amplifier minimizes the total noise figure of the whole receiver system. From the focal line, the signal is transferred by coaxial cable to the outdoor cabin located at the base of the antennas; the signal is filtered again and then handled by a vector modulator, required to form the beam. The next steps are the signal down conversion in base band, the low pass filtering and digitalization. The digital outputs are formatted following the Ethernet protocol and transferred through digital optical transceivers via optical mono-modal fibre link to the processing room for further elaborations, after an electrical back conversion. In this architecture the local oscillator, the digital clock control and the synchronization signals are sent from the processing room to the outdoor reference cabin. The power supply is sent to the cabins and to the devices placed on the antenna focal lines through the coaxial cable.
In the second receiver chain architecture, named analogue optical link solution, the signal is sent from the front ends installed on the focal lines to the processing room via optical analog link. In Fig. 2 we can see the main transmission and control signals according the second solution always for a chain of Northern Cross N-S arm. In particular we can observe that the external links are composed by only mono-modal optical fibre for signal transmission and simple cables for power supply. The use of an optical transmitter (laser) on the antenna focal lines needs of an analog fibre link in order to transport the signal to the data processing room. In this solution, in order to remove the high noise figure due to the laser and to increase the gain before optical link, in the focal line subsystems it is necessary to introduce three low noise amplifier stages and two pass-band filters between them. This optical signal reaches the processing room where we can find an optical receiver, an analogue receiver and a VIIP (Very Inexpensive Industrial Prototype) board. The VIIP board is made up by two high resolution ADCs, which digitize the signals coming from two analog inputs, one FPGA, which implements a DDC (Digital Down Converter) and a PFB (Polyphase Filter Bank). The main advantage of this architecture is that the local oscillator and the synchronism signals are distributed only in the main data processing room.

By a comparison between such solutions, it appears that re $-\int$ ceiver chain performances based on analogue optical link seem better in terms of:

- potential wider bandwidth for further upgrading (up to $2.5 \mathrm{GHz}$ ),

- electrical insulation,

- immunity to electromagnetic interferences.

Even from the reliability and maintainability point of view, the architecture of the analogue optical link solution seems to be stressed by lower environmental solicitation levels being more devices in a sheltered environment; at the same time the architecture is characterized by easier maintenance because more devices are placed in an easily reachable area.

\section{Approach for the system reliability}

The reliability is the ability, or the probability, of an item to maintain the required functions in the time, under given conditions (IEC 1990). In the case under study, we can consider the entire radioreceiver equipment as the item. It's important to correlate the reliability to the failure rate $\lambda(t)$. Considering a generic time interval $\Delta_{t}$, the failure rate is defined as the probability of a failure occurrence in the interval $[t, t+\Delta t]$, given that the element or the system was new at $t=0$ and did not fail in $(0, t]$ (Shoman 1990). In order to consider the worst case of reliability prediction, we assume that all the devices of the receiver chains are in reliability series configuration with random and indipendent failures. In such hypothesis the corresponding reliability mathematical model is:

$$
R_{s}(t)=\prod_{i=1}^{n} e^{-\lambda_{i} t}=e^{-\lambda_{s} t}
$$


where $\lambda_{i}$ is the failure rate of each devices and $\lambda_{s}$ the failure rate of the system:

$$
\lambda_{s}=\sum_{i=1}^{n} \lambda_{i}, \text { in failures } * \text { hours }^{-1}
$$

We will assume the expectation of the operating time between failures, known as Mean Time Between Failure (MTBF), as a measure of the reliability performance of the equipment. With the assumptions above, we have:

$$
\operatorname{MTBF}=\frac{1}{\lambda_{s}}, \text { in hours }
$$

The evaluation of such performances can be implemented using prediction technique. In this case, referring to Eq. 2, we can calculate the failure rates $\lambda_{i}$ of all elements of the chainstructure and consequently, the MTBF value of the system. With this theoretical approach we are comparing two different design architectures, in our case, the digital optical link and analogue optical link solutions. We implemented a reliability prediction with Part-stress Analysis method by means of the Military HandBook 217 FN2 (U.S.A. Department of Defence 1991; IEEE Std 2003). In particular, for discrete components, the failure rate $\lambda$ under operating conditions is given by the following model:

$$
\lambda=f\left(\lambda_{b}, \pi_{i}\right)
$$

where $\lambda_{b}$ denotes the basic failure rate and $\pi_{i}$ are correction factors, for example, in function of operating environment, temperature, electrical stress, quality production and design technology. Regarding the operating environmental, we consider the following three different environments defined in the MILHDBK-217 FN2 for standard application:

- Ground Benign (GB), fixed weather protected environment;

- Ground Fixed (GF), fixed but non weather protected environment;

- Ground Mobile (GM), characterized by mechanical stresses and severe thermal gradients.

In the follows we present the results, the evaluation of MTBF for the different design solutions, obtained with the predictive analysis approach. The digital optical link solution (Fig. 1) is made up of three stages and we can consider three different operative environments. The analogue optical link solution (Fig. 2), instead, is constituted only by two stages being electronics in outdoor cabin removed. In both cases a reliability analysis was performed from the front-end subsystems up to Back-end input. As operative environments we assumed Ground Mobile (GM) for the antenna focal line subsystems, Ground Fixed Uncontrolled (GF) for the subsystems placed in the cabin and Ground Benign (GB) for the devices in the data processing room. Moreover, the operative temperature was chosen $40{ }^{\circ} \mathrm{C}$ for the GM environment, $30{ }^{\circ} \mathrm{C}$ for the $\mathrm{GF}$ and $25{ }^{\circ} \mathrm{C}$ for the GB. We supposed that the system working $24 \mathrm{~h}$ on $24 \mathrm{~h}$ so duty cycle equal to $100 \%$ is assumed. According to Eq. 1 and relative hypothesis, the results of the reliability prediction so obtained are shown in Table 1 and 2 for the two architectures. In particular for each subsystem and devices constituting the architecture the corresponding MTBF is summarised.
Table 1: Reliability prediction of digital optical link architecture

\begin{tabular}{|l|c|}
\hline Assembly name & MTBF $[\mathrm{h}]$ \\
\hline \hline DIGITAL OPTICAL LINK SOLUTION & 10624 \\
\hline Focal line Subsystems & 97444 \\
\hline Front End & 97444 \\
\hline Cabin Subsystems & 15658 \\
\hline Receiver & 607669 \\
\hline VIIP & 60268 \\
\hline Ethernet & 177766 \\
\hline Optical TX & 25000 \\
\hline Data Processing Room Subsystems & 50000 \\
\hline Optical RX & 50000 \\
\hline
\end{tabular}

Table 2: Reliability prediction of Analogue Optical Link Architecture

\begin{tabular}{|l|c|}
\hline Assembly name & MTBF $[\mathrm{h}]$ \\
\hline \hline ANALOGUE OPTICAL LINK SOLUTION & 37187 \\
\hline Focal line Subsystems & 44600 \\
\hline Front End & 97444 \\
\hline 3rd Stage & 986362 \\
\hline Optical TX & 89721 \\
\hline Data Processing Room Subsystems & 15658 \\
\hline Optical RX & 515615 \\
\hline Receiver & 2336591 \\
\hline VIIP & 475694 \\
\hline
\end{tabular}

According to Eq. 2 and 3 we obtained a value of the failure rate $\lambda_{s}$ equal to $94.125 * 10^{-6} \mathrm{~h}^{-1}$ for the digital optical link solution; consequently we can calculate an MTBF of 10624 hours, about 1.2 years of continuous operation without maintenance actions. On the same hypothesis, for analogue optical link configuration, we have $\lambda_{s}$ equal to $26.891 * 10^{-6} \mathrm{~h}^{-1}$ and MTBF of 37178 hours, about 4.2 years. On the basis of the architectural advantages and reliability prediction results, we decided to implement, for the re-instrumentation of the radioreceiver chain of the Northern Cross, the analog optical link solution. After the implementation of the radioreceiver chain we decided to monitor the real thermal stress variation on the subsystem placed on the focal line, that appears the most critical by the environmental point of view. In fact, it is well known that failure rate of electronic equipments depends overall from the temperature (Lall et al. 1997).

\section{Thermal characterization of the critical subsystem}

It is interesting to observe that, from a climatic-environmental point of view, the most stressed devices could be those placed on focal line, the front-end with three stages of amplification and the optical transmitter. So we decided to monitor the 


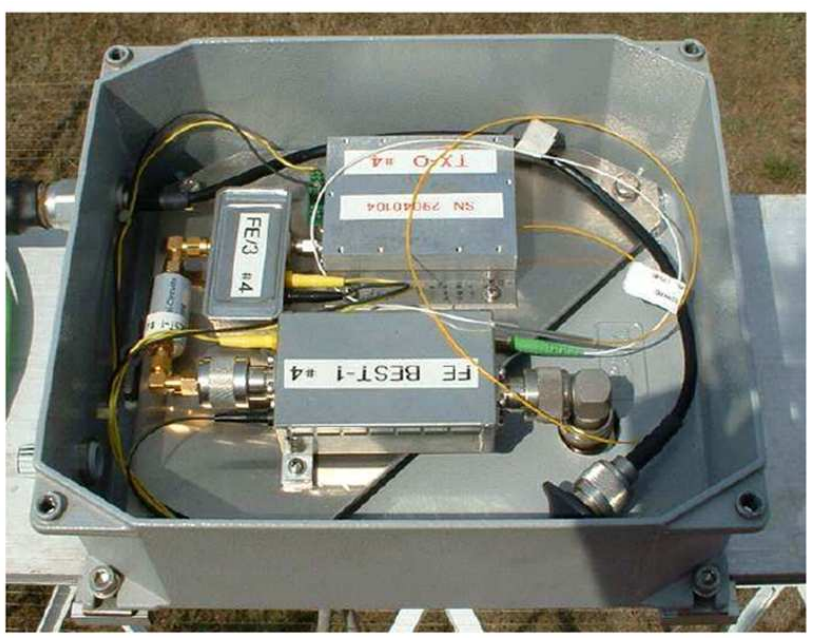

Fig. 3: BEST-1: one of the four receiver boxes installed on the antenna

real thermal variation for the solution implemented for the reinstrumentation of the Northern Cross.

A methodology to reproduce slow variations of operative temperature of electronic devices, and to measure the induced effects on them, is presented. That method has been conceived in order to test and characterise the new receivers developed for Northern Cross radio telescope. All the instruments of the measurement system are controlled by a PC (i.e. initialization, calibration and data acquisition) in order to obtain a new thermal characterization method of electronic devices, complementary to the most common one, where the devices are measured at different, but steady, operative temperatures. In conventional radio telescopes, the receivers are located in well protected and controlled environments. In particular their operative temperature is kept constant from the low noise amplifier, which often works at cryogenic temperatures, to the back-end. Moreover through this remote control it is always possible, even during an observation, to perform their calibration by means of injections of well know amounts of noise, typically just after the antenna output and, in any case, before the low noise amplifier. Such way is not feasible when the receivers are arranged in array configuration with a large number of elements and/or they are distributed over a wide area (i.e. not in the focus of a dish). This is the typical situation of the Aperture Array technology (AA), which is probably the most promising and versatile one, and, over all, the only that can fully satisfy the strong astronomers' requirements in the SKA low frequency (i.e. less than $1 \mathrm{GHz}$ ) band. The radioreceiver chain implemented on the Northern Cross (BEST) is a modular technological demonstrator composed by 3 blocks with different dimensions and characteristics (i.e. collective area, number of receivers, frequency band). BEST receivers are located inside waterproof boxes and are composed by a three stages low noise front end and an optical transmitter, in order to transport the RF signals $(16 \mathrm{MHz}$ centred at $408 \mathrm{MHz}$ ) directly in a central protected and shielded receiver room, located inside the main building. There, signals are down converted, digitised and finally processed by a powerful, FPGA based, digital back end. In order to know the ef-

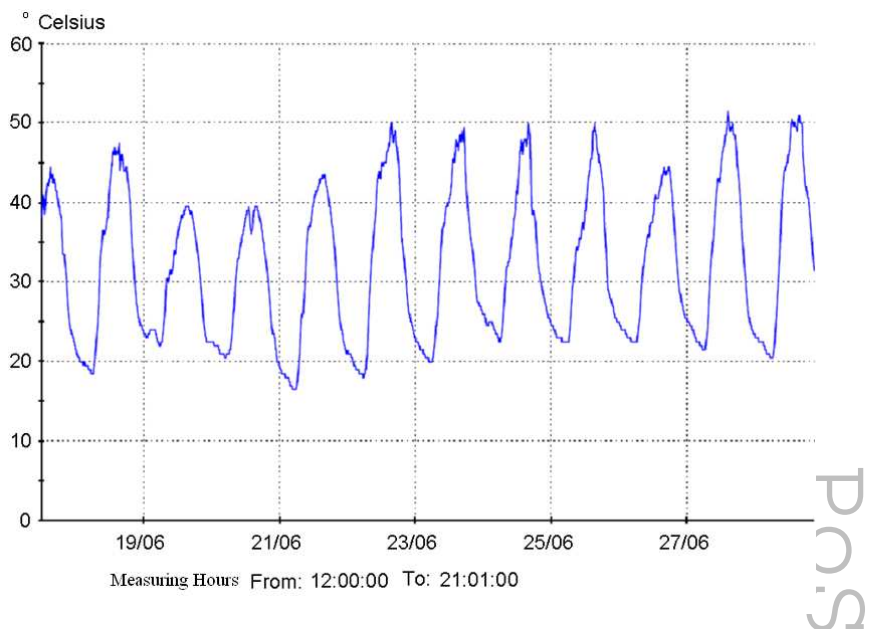

Fig. 4: Typical temperature variation of the receiver boxes in summer time

fective thermal influence on the focal line devices and conse quently to implement a more specific reliability prediction of the radioreceiver chain in function of the real thermal stress, we monitored the temperature inside the boxes installed on the focal lines of the Northern Cross radio telescope, both in summer and winter time. The measured thermal excursions are from $+50{ }^{\circ} \mathrm{C}$ to $+20^{\circ} \mathrm{C}$, in about 12 hours, from day to night, and vice versa from $+20{ }^{\circ} \mathrm{C}$ to $+50{ }^{\circ} \mathrm{C}$ from night to day during summer (Fig. 4) or from $10{ }^{\circ} \mathrm{C}$ to $-5{ }^{\circ} \mathrm{C}$ in winter.

This thermal monitoring it was useful also in order to understand if it possible to compensate gain and phase variations of receivers, induced by external temperature variations (i.e. day night, sunny to cloudy and/or rainy), only at back-end stage (i.e. with periodic observations of strong well known ra-

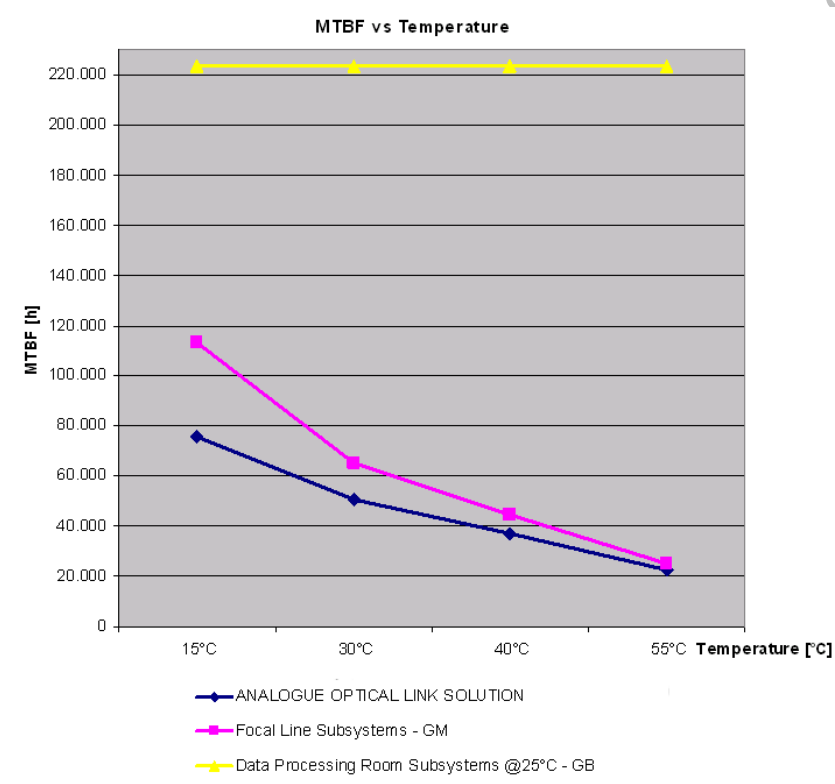

Fig. 5: Evaluation of the MTBF for the analogue optical solution vs. temperature 
Table 3: Reliability prediction of the Analogue Optical Link solution in function of thermal stress variation

\begin{tabular}{|l|c|c|c|c|}
\hline \hline \multirow{2}{*}{$\begin{array}{l}\text { Assembly name } \\
\text { Subsyl line }\end{array}$} & \multicolumn{4}{|c|}{ MTBF $[\mathrm{h}]$} \\
\hline & GM@ $15^{\circ} \mathrm{C}$ & GM@ $30^{\circ} \mathrm{C}$ & GM@ $40{ }^{\circ} \mathrm{C}$ & GM@ $55^{\circ} \mathrm{C}$ \\
\hline $\begin{array}{l}\text { Data Processing } \\
\text { Room Subsystems }\end{array}$ & $113186 \mathrm{~h}$ & $65176 \mathrm{~h}$ & $44600 \mathrm{~h}$ & $25288 \mathrm{~h}$ \\
\cline { 2 - 5 } $\begin{array}{l}\text { ANALAGUE } \\
\text { OPTICAL LINK } \\
\text { SOLUTION }\end{array}$ & $75652 \mathrm{~h}$ & $50653 \mathrm{~h}$ & $37187 \mathrm{~h}$ & $22757 \mathrm{~h}$ \\
\hline
\end{tabular}

dio sources called "calibrators"). To this aim, in the receiver design phase, a deep thermal characterization has been carried out, measuring the gain variations both of front-end and optical transmitter, at different, but steady, operative temperatures in those range (Montebugnoli et al. 2005). In other worlds it was investigating if it is possible to avoid any active temperature control system and any hardware calibration system. On the basis of the thermal monitoring we implemented a new reliability prediction, only for the architecture solution implemented, in order to evaluate possible degradation of the critical subsystem of the entire chain. The reliability prediction was done according to the same prediction hypothesis presented in the section 2. In Table 3 is shown the reliability prediction of the focal line subsystem, varying the operative temperature. In this case the reliability prediction for the data processing room subsystem is assumed fixed, thanks to their operating environment. Consequently we evaluated the MTBF for the entire receiver chain at different temperature. Fig. 5 shows the plots of MTBF subsystems and system versus temperature. The yellow line is the constant MTBF of the subsystem placed in the Ground Fixed, the violet line plots the behaviour of the subsystem placed on the focal line in Ground Mobile environment and finally the blue line is the plot of the consequent behaviour of the entire receiver system. It is interesting to observe that the MTBF of the entire receiver chain system decreases of about $26,6 \%$ if the devices placed on the focal line work at $40{ }^{\circ} \mathrm{C}$ instead at $30{ }^{\circ} \mathrm{C}$ and of about $55 \%$ if their operative temperature increases at $55^{\circ} \mathrm{C}$. In order to further investigate the reliability of the thermal critical devices and their possible deficiencies in the design or production process by means of an experimental approach, we suggest implementing environmental screenings and life tests. In particular we propose a Z/AD test (IEC 1998), in which the stresses are temperature and humidity, properly planned considering the right stress level and physico-chemical model of degradation. This kind of combined solicitation is useful to detect defects by "breathing" as distinct from absorption of moisture and it differs from other damp heat cycles for a greater severity; in fact, there are a higher number of temperature variations in a given time, a greater cyclic temperature range, a higher cyclic rate of change of temperature and a number of excursions to sub-zero temperatures. From the outcomes of compliance preliminary tests we just observed criticalities after the Temperature and Humidity cyclic test, ev- idencing clearly the necessity to optimize some elements of assemblage of the receiver chain (Catelani et al. 2009a,b).

\section{Conclusions}

Different architectures of radioreceiver chains have been investigated and compared in order to perform the BEST goals and, in general, the main SKA related technology requirements: low cost, high performances and reliability. These experimen tal studies are leading to the design of receiver chains suitable for a square kilometer array radiotelescope and for other complex applications designed for reliability. In fact, the approach described here has general validity and could be applicable to every kind of complex electronic or mechatronic systems. In this work a reliability performance evaluation was implemented on different radioreceiver chains solution of the Northern Cross Radiotelescope. The results so obtained proved that environmental conditions where the system is located play a fundamental role from the reliability point of view. The analogue optical link configuration radioreceiver chain showed a higher MTBF so as to guarantee to the new Northern Cross a greater reliability, with the consequent less probability of fault and lower maintenance costs. These results were very important for the re-engineering of Northern Cross, as a SKA test bed, but they will be very valuable also in the proper design of the new generation SKA radiotelescope. In fact the dimension and the cost of the SKA radiotelescope recommend to deep the reliability of the instruments and devices that will be implemented.

\section{References}

Bolli, P., F. Perini, S. Montebugnoli, G. Pelosi, S. Poppi, "Basic Element for Square Kilometer Array Training (BEST): Evaluation of the Antenna Noise Temperature, Antennas and Propagation Magazine," IEEE, Vol. 50, Issue: 2, April 2008, Page(s):58-65 ISSN: 1045-9243

Catelani, M., V. L. Scarano, R. Singuaroli, S. Montebugnoli, G. Bianchi, F. Perini, I. Trotta, "Northern Cross Radiotelescope: test and measurements of reliability performance on radioreceiver chains," IEEE Transactions on Instrumentation and Measurement; Vol 58, Issue 10, Oct. 2009 Page(s):3769 3777, ISSN: 0018-9456

Catelani, M., V. L. Scarano, R. Singuaroli, S. Montebugnoli, G. Bianchi, F. Perini, "Environmental Testing: Behaviour 
of a Radioreceiver Chain Front-end," Metrology And Measurement Systems, Volume XVI - No 1/2009, ISSN 0860-8229

Dewdney, P.E., Hall, P.J., Schilizzi, R.T., Lazio, T.J.W., “The Square Kilometre Array," Proceedings of the IEEE, Vol. 97, No. 8, Aug. 2009 pp. 1482-1496, ISSN: 0018-9219

Hall, P.J., "The Square Kilometre Array: An Engineering Perspective," Experimental Astronomy, Springer Netherlands, Volume 17, Numbers 1-3 / June, 2004, Page(s): 5-16, ISSN: 0922-6435 (Print) 1572-9508 (Online) IEC 50 (191) "International Standard: International Electrotechnical vocabulary - Chapter 191 - Dependability and Quality of Source," International Electrotechnical Commission, Genève, Swisse - 1990

IEC 60068, "Basic environmental testing procedures," December 1998

IEEE Std, "IEEE Guide for Selecting and Using Reliability Prediction Based on IEEE" 1413, Feb 19, 2003

Lall, P., M. G. Pecht, E. Hakim, "Influence of temperature on microelectronics and system reliability: A physics of failure approach," CRC Press, New York, NY (1997)

Montebugnoli, S., G. Bianchi, C. Bortolotti, A. Cattani, E. Ghelfi, A. Maccaferri, F. Perini, J. Roda, M. Roma, G. Zacchiroli, L. Zoni, "Re-instrumentation of the Northern Cross Radio Telescope for a SKA Cylindrical Concentrator Based Test Bed," Proceedings of 34th European Microwave Conference - Amsterdam, 2004, Page(s): 1537- 1540

Montebugnoli, S., M. Boschi, F. Perini, P. Faccin, G. Brunori, E. Pirazzini, "Large antenna array remoting using radioover-fiber techniques for radio astronomical application," Microwave and Optical Technology Letters, Vol.46, No.1, July 52005

Montebugnoli, S., et al., "BEST (Basic Element for SKA Training)," available via http: //www.med.ira.inaf.it/ BEST/index_en.htm

Shoman, M.L., "Probabilistic reliability: an engineering approach," second edition, Krieger Pub. Company, 1990

U.S.A. Department of Defence, "Military Handbook Reliability Prediction of Electronic Equipment," MILHDBK-217F, 1991 (and later versions)

van Ardenne, A., "Concepts of the Square Kilometre Array; toward the new generation radio telescopes," proceedings of IEEE Antennas and Propagation Society International Symposium, Salt Lake City, UT, USA, July 2000. Vol. 1, page(s): 158-161 